\title{
Automatic Sensor Management: challenges and solutions
}

\author{
Tanja van Valkenburg- van Haarst ${ }^{1,2}$, Wilbert van Norden ${ }^{1,3}$, Fok Bolderheij ${ }^{1}$ \\ ${ }^{1}$ CAMS- Force Vision, P.O. Box 10.000, MPC 10A, 1780 CA Den Helder, the Netherlands. \\ ${ }^{2}$ University of Amsterdam, P.O. Box 19268, 1000 GG Amsterdam, the Netherlands \\ ${ }^{3}$ Delft University of Technology, Mekelweg 4, 2628 CD Delft, the Netherlands
}

\begin{abstract}
Due to technical advances and the changing political environment sensor management has become increasingly knowledge intensive. Aboard navy ships however, we see a decrease of available knowledge, both quantitative and qualitative. This growing discrepancy drives the need for automation of sensor management. Since the goal of sensor deployment is to have a complete and accurate operational picture relative to the mission we propose a three-stage sensor manager, where sensor task requests are generated based on the uncertainty in the (expected) objects' attributes. These tasks are assigned to available and suited sensors, which in turn are fine-tuned for the task at hand. When trying to reduce the uncertainty in the classification solution one must first define how the classification process actually works. We discuss why the classification process needs to be automated as well and show how such classification algorithms will most likely work in the future.
\end{abstract}

Keywords: Sensor Management, Optical Sensors, Command and Control

\section{INTRODUCTION}

Managing the sensor systems onboard modern naval vessels requires an increasing amount of technical knowledge due to the fact that these vessels are equipped with state of the art sensor systems that provide more functionality and more accurate information at the cost of more complex control mechanisms. Furthermore, the shift of operational areas to littoral waters with often-dense civil traffic and rapidly changing geographical and meteorological conditions demands a much more dynamic adaptation of the sensor settings in comparison with the more stable environment of traditional operational areas like the Atlantic Ocean. The shrinking of the defense budgets on the other hand give rise to a demand for crew reduction, shorter education times and less training opportunities causing a reduction of the synergy created within teams of operators and the knowledge and experience of individual operators.

From these observations can be deduced that sensor management requires an increasing amount of both operational and technical knowledge while the available amount of these types of knowledge is decreasing. The consequences of incorrect sensor management may however be severe: if sensor systems fail to detect threatening objects, platforms may be incapacitated or even neutralized and consequently mission objectives will not be met. Therefore research has to be executed to model and capture sensor management related knowledge in order to support the deployment of complex sensor systems.

An additional challenge is presented by the integration of optical sensors like infrared (IR) sensors and High Definition (HD) camera's in Command and Control (C2) systems, as these sensors provide a different type of information in comparison with the data provided by 'traditional' sensors like radar and Identification Friend or Foe (IFF). The available sensors therefore have to be described in terms of the type of information they provide and how this information contributes to the compilation of the Operational Picture (OP) as this picture forms basis for decision making processes and subsequent actions. The sensor management process now has to deploy the available sensors in such a way that the OP is as complete and accurate as possible given the available sensor systems.

In Section 2 of this paper the purpose of sensor management is explained, related performance indicators are derived and sensor management principles are formulated. Section 3 describes how these principles can be used to construct a threestage sensor manager and how this sensor manager uses the information compiled in the OP by C2 processes, to deploy the sensors. Section 4 explains how optical systems can contribute in the compilation of an OP. In Section 5 conclusions are drawn and the required future work is discussed.

Optics and Photonics in Global Homeland Security IV, edited by Craig S. Halvorson, Daniel Lehrfeld, Theodore T. Saito Proc. of SPIE Vol. 6945, 694511, (2008) · 0277-786X/08/\$18 - doi: 10.1117/12.777607 


\section{SENSOR MANAGEMENT ISSUES}

As already brought forward in the previous section, sensor management is currently executed by operators, who translate the operational requirements of a mission into technical sensor settings while taking operational, environmental and political constraints like Rules Of Engagement (ROEs) and Emission Control (EMCON) plans into account. Because these technical controls are sensor specific and may be implemented in hardware or are made available by Graphical User Interfaces (GUIs), an operator must be familiar with the meaning of each setting and how a change of setting affects the performance of the sensor. In the previous section was also mentioned that the operator must be aware of the complementary properties of the various sensors and actually has to consider the control of the complete sensor suite as opposed to the setting each sensor individually.

Specifically looking at the use of electro-optical sensor systems during a mission, it can be stated that an operator uses the cameras to visually detect and classify objects in the ship's surrounding. Classification is achieved by comparing the information provided by the sensor system to prior knowledge about the expected targets while compensating for the environmental influences. Based on the performance of the sensor systems, the range of the object of interest and the operational constraints for the mission, the sensor system that will be used to classify the object is selected.

In order to classify an object by means of electro-optical sensor systems, the operator compares the silhouette as presented by the electro-optical sensor system with pictures of the silhouettes of the expected objects. To compare the silhouettes, the operator mainly focuses on distinct points of the object while taking his prior-knowledge of the capabilities and limitations of the object into account. The object is considered classified when the picture and the silhouette match. When no match can be found and the object is assumed to be relevant to the mission, the crew will put more effort into the classification of the object by deploying other sensor systems or by maneuvering the platform nearer to the object. In order to support the operator in the classification process, the sensor has to provide the most detailed picture possible that shows a sufficient number of classification features. The sensor therefore has to be optimized for this specific classification task.

The observation that system-specific sensor management is a complex task that requires extensive operational and technical knowledge is recognized in literature and various papers can be found that propose methods and algorithms to support this task. Strömberg, et al. [1] have conducted a literature survey that presents an overview of relevant principles and methods concerning sensor management. Most of the methods reviewed by them provide a technical, sensororiented approach, striving for optimal sensor settings, but leave the translation of the operational requirements into technical sensor settings to the operator and therefore do not provide a solution to the identified problem. McIntyre and Hintz have compiled a Comprehensive Approach to Sensor management, consisting of three papers [2], [3], [4], that describes a survey of modern sensor management systems, a new hierarchical model and goal lattices. In their first paper, [2], they present the concept of the sensor management process and recognize sensor management as a process that contributes to the realization of the mission goals; how this may be achieved is however not directly clear.

Interviews with operational experts [5] revealed that two important requirements with respect to the compilation of the OP have to be met:

1. the OP must be complete;

2. the OP must be accurate.

This means that the deployment of the sensor systems must be aimed at satisfying these requirements. Bolderheij and Van Genderen [6] argue that these requirements can be met by constructing the OP from objects that represent the mission-relevant elements in the environment. They state that the OP can be considered complete if each relevant element in the environment is represented by at least one (preferably by only one) object in the OP and that the accuracy of the OP can be pursued by reducing the uncertainty that belongs to the attributes of the object. To maintain the completeness of the OP, sensor systems have to be deployed to search the environment for the presence of these elements while the accuracy can be ensured by tracking, classifying and identifying them. To initiate surveillance, the expected threat in the operational area is analyzed during the planning stage of a mission, and modeled as expected objects that are inserted in the OP. 


\section{A THREE-STAGE SENSOR MANAGER EMBEDDED IN THE C2 PROCESS}

Taking the OP as a starting point, Bolderheij and Van Genderen [6] have developed a sensor manager that distributes available sensor resources in order to compile an OP that satisfies the requirements formulated in the previous section. This sensor manager first inspects the attributes of each object in the OP with respect to the amount of uncertainty that is related to its attributes and assigns sensor tasks to reduce this uncertainty. The sensor manager then selects the most appropriate sensor from the set of available sensors and finally tunes the sensor for this specific task.

In Fig. 1 is shown how the OP acts as the central element among the $\mathrm{C} 2$ processes, amongst which the three-stage sensor manager is placed. It can be seen as a virtual blackboard on which the $\mathrm{C} 2$ processes write their information and/or from which they read their information. It may also be implemented as a market place where agents update or retrieve the information contained in the object attributes.

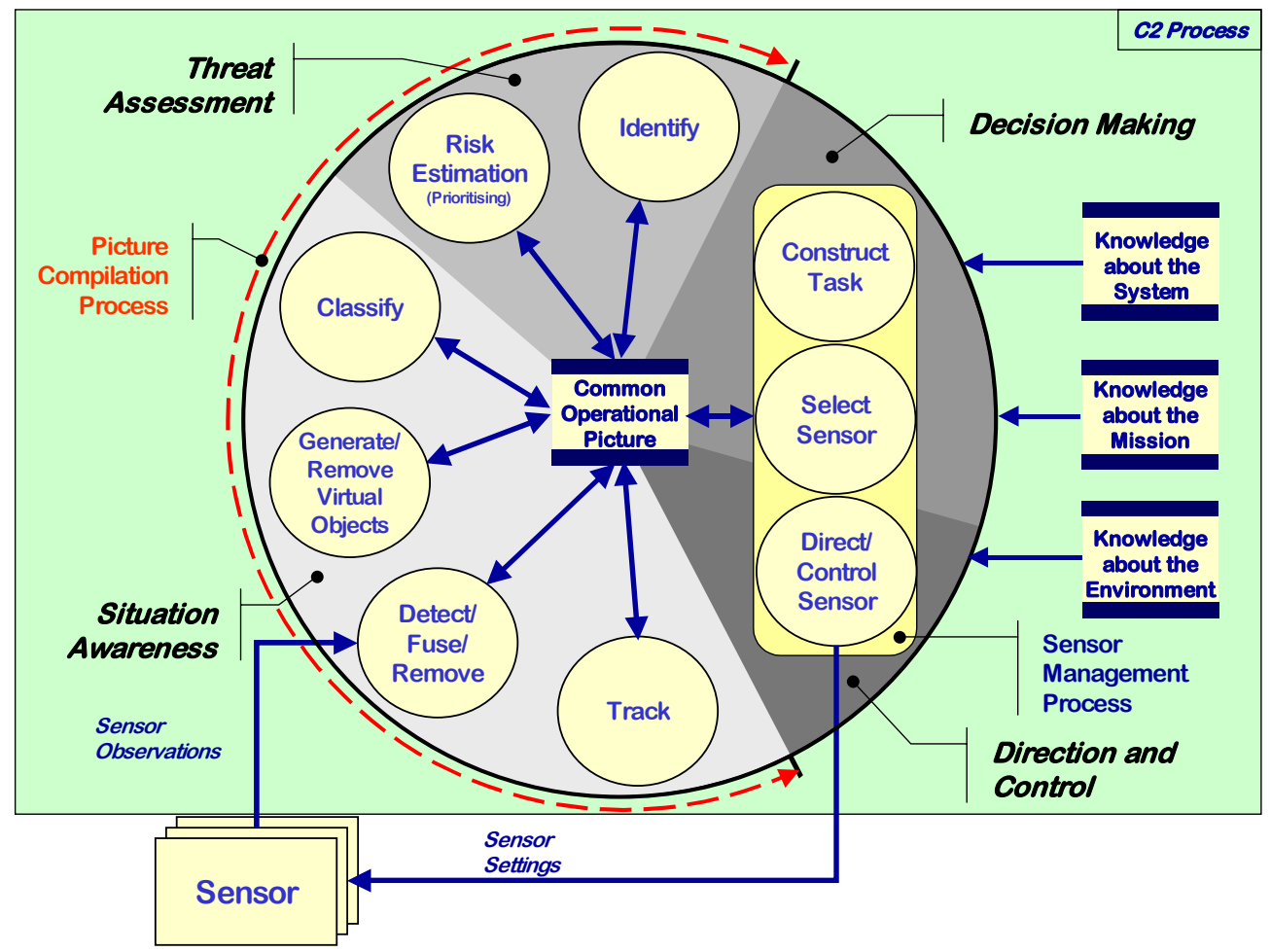

Fig. 1. The object-centric $\mathrm{C} 2$ process with embedded sensor manager

Generally speaking, the sensor resources are not unlimited available and the allocation has to be prioritized. Therefore a prioritizing mechanism was developed that estimates the risk posed by an interaction with an object based on the presumed lethality of the object and the probability of occurrence of the interaction.

In Fig. 1 the three-stage sensor manager is shown in the context of the overall C2 process. Through the sensors placed in the lower left corner, a control cycle over those sensors can already be seen. Fig. 2 shows the three-stage sensor manager but now directly in the context of a sensor control cycle. The first two stages of sensor management are highlighted in Fig. 2 since they both are the main focus for now.

\subsection{Stage 1: Constructing a sensor task}

In [5] and [6] the notion of risk was used as a prioritization criterion for the scheduling of sensor tasks. How such tasks were made however, was not discussed. Here we will describe how tasks are generated, utilizing the risk calculation given by Bolderheij. Each object in the environment has $n$ attributes, $\mu_{1}, \ldots \mu_{n}$, e.g., the attributes: position, speed, acceleration, class or type, and identity. Risk, denoted $R$, is calculated as a function of these attributes and our current mission, denoted $M$. In formula this statement translates to: $R=f\left(\mu_{1}, \ldots, \mu_{n} \mid M\right)$. Of course, some of the attributes have 
an amount of uncertainty, denoted $\sigma_{1} \ldots \sigma_{n}$. Where the sensor manager previously looked at the risk calculated based on the mean values of attributes, we now expand this by sequentially varying the different attributes to compute the uncertainty in risk, denoted $\sigma_{R}$. By sequentially varying the uncertainties we can also see how each $i$-th attributeuncertainty, $\sigma_{i}$, contributes to the uncertainty in risk, denoted $\sigma_{R, i}$. Equation (1) shows how the risk uncertainty is calculated when risk is a function of three attributes, $\mathrm{R}=f\left(\mu_{1}, \mu_{2}, \mu_{3}\right)$. The exact formula to calculate risk is of little importance here since we only describe the conceptual design. The formulation of risk that we use can be found in [6]. Since the computation of risk uses Dynamic Bayesian Networks, the mission variables are embedded in the risk function, which explains why the mission is no longer seen in the formulas presented here.

$$
\begin{gathered}
\sigma_{R}=\sqrt{\sigma_{R, 1}{ }^{2}+\sigma_{R, 2}{ }^{2}+\sigma_{R, 3}{ }^{2}} \\
\sigma_{R, 1}=f\left(\left(\mu_{1}+\sigma_{1}\right), \mu_{2}, \mu_{3}\right)-R \\
\sigma_{R, 2}=f\left(\mu_{1},\left(\mu_{2}+\sigma_{2}\right), \mu_{3}\right)-R \\
\sigma_{R, 3}=f\left(\mu_{1}, \mu_{2},\left(\mu_{3}+\sigma_{3}\right)\right)-R
\end{gathered}
$$

When $\sigma_{R, i}$ and/or $\sigma_{i}$ exceeds a user-defined threshold, a task is requested to minimize the uncertainty by executing a sensor task of type $T$. Using this methodology we can reason which attribute uncertainty needs to be reduced, by choosing the appropriate type of sensor task to perform. And we can also compute the amount of uncertainty that needs to be reduced for that attribute, denoted $\xi$. These thresholds can be determined and fine-tuned in simulations in the planning stage of a mission thus enlarging the possibility of mission success.

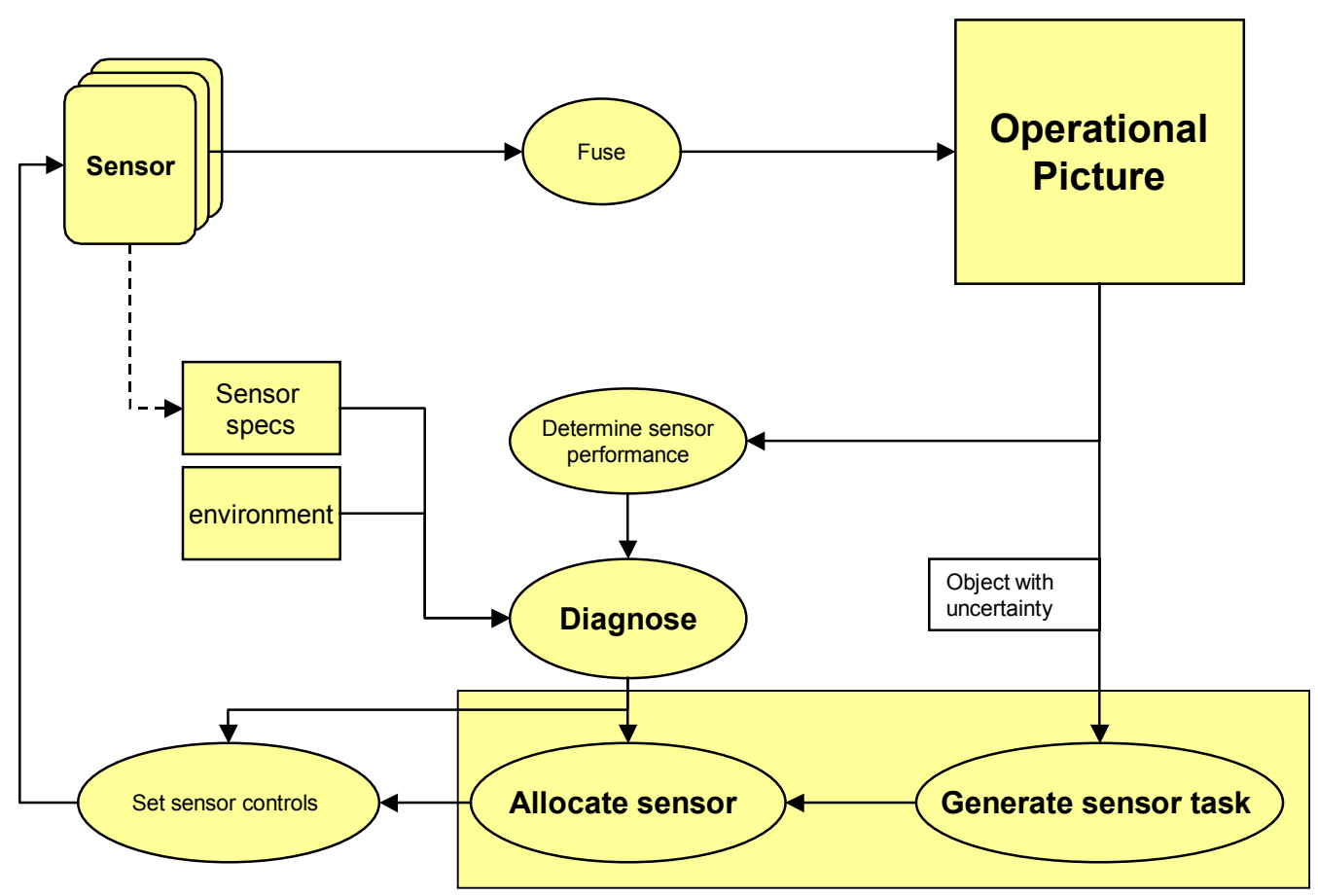

Fig. 2. The three-stage sensor manager placed within the sensor control cycle where uncertainty on object information is used to generate sensor function requests and sensor performance determines the sensor allocation. 
The object's kinematics and current position define the area $A$ where the contact is expected to be. The upper bound of the risk $\left(R+\sigma_{R}\right)$ defines the priority, $\Phi$, of the task. A due date of the task, $t_{d d}$ is added to ensure that $\sigma_{R}$ does not exceed mission dependent thresholds. Based on these definitions a mapping formula, given by equation (2), can be constructed.

$$
\operatorname{contact}\left([\bar{\mu}, \bar{\sigma}],\left[R, \sigma_{R}\right]\right) \rightarrow \operatorname{task}\left(T, A, \xi, t_{d d}, \Phi\right)
$$

\subsection{Stage 2: Assigning a sensor}

When a task is generated it has to be executed by one of the available resources and preferably by the best-suited resource. Although this might seem straightforward, it is not. The problem for one multifunction radar has been shown to be NP-hard by Thaens in [7]. Adding more resources will certainly not make the problem easier. Of course, when a task

can only be executed on one resource, the allocation problem is already solved. If this is not the case, the problem becomes a multi-criteria scheduling problem of which an overview can be found in T'Kindt and Billaut [8].

To find a scheduling methodology we first have to look at the various criteria that are involved in the sensor allocation problem. First criterion can be implemented relatively simple for the time being: is the object in range of a sensor given the specifications. A model of the sensor and the current environment are needed to determine the detection probability of sensor $S$ for the object at which the requested sensor task $j$ is directed, denoted $P_{s, j}$, and the sensor's detection accuracy, denoted $\zeta_{s, j}$. A second criterion is the operator bias for a certain sensor. Such bias is modelled in this methodology and is denoted by $\zeta_{u, T}$. This factor could also be used to disable certain functionalities of sensors, e.g. due to malfunctioning or during maintenance.

A third criterion is the availability, $\tau$, of the sensor for the specific task under consideration. Availability can mean 1) that the sensor has nothing to do at the moment or 2) the user has authorized the use of this sensor for that specific task. The first is denoted by $\tau_{s}$ and is computed with equation (3). The latter is defined as: $\tau_{u, s}=1$, off: $\tau_{u, s}=0$, or any other value $0<\tau_{u, s}<1$ to indicate degraded performance of the entire sensor.

$$
\tau_{s}=\left\{\begin{array}{lc}
t_{c}+t_{p} \leq t_{d d}: & \frac{1}{t_{c}+t_{p}-t_{d d}} \\
t_{c}+t_{p}>t_{d d}: & 0
\end{array}\right.
$$

In equation (3) the time a sensor needs to perform a task is denoted by $t_{p}$ and the current time is denoted by $t_{c}$. Including these time factors ensures that it becomes increasingly important to execute a task as its deadline approaches.

Trying to solve the allocation problem, means we have to combine all these factors into one formula. The result is equation (4), which computes the effectiveness of a sensor for all tasks of type $T$ that currently need to be allocated, denoted $E_{T, s}$ where $j=1 \ldots J$.

$$
E_{T, s}=\tau_{u} \cdot \zeta_{u, T} \cdot \sum_{j=1}^{J}\left(\frac{\zeta_{s, j}}{\xi_{j}} \cdot \tau_{s, j} \cdot P_{s, j} \cdot \Phi_{j}\right)
$$

The optimization of equation (4) can be done in several ways. Van Norden et.al. [9] discuss the fuzzy Lyapunov approach (based on a solution to the job-shop scheduling problem from Margialot and Langholz, [10]) as well as an online evolutionary algorithm. Another approach is using hybrid metaheuristics as described in [11] and [12]. Currently, the preference is to use the fuzzy Lyapunov approach because this has a good trade-off between performance and computation time as can be see in aforementioned references. The formula presented in [9] however is replaced by equation (4). 
The resulting algorithm is based on making buffers for each different task type. By calculating the effectiveness of the sensors for each of these buffers, choices can be made in the allocation process. E.g., suppose that a task of type A can be executed on either sensor $\mathrm{X}$ or sensor $\mathrm{Y}$. Using the effectiveness formula this task will be allocated to sensor $\mathrm{X}$ if $\mathrm{E}_{\mathrm{A}, \mathrm{X}}>$ $\mathrm{E}_{\mathrm{A}, \mathrm{Y}}$. Because each task has a certain contribution to the buffer weight it can also be used as a scheduling methodology. More details about fuzzy Lyapunov for sensor scheduling can be found in [12].

\subsection{Stage 3: Tuning the sensor}

Through the objects in our environment, tasks are created and assigned to the most suited sensor at that time. The third stage of the sensor manager is setting the sensor optimally for the task at hand given the current environment and the mission restrictions.

For some sensors this is easy: an electro-optical sensor is directed to the contact and zooms according to the distance. For a radar system however, calculating the optimal waveform, pulse repetition rate and frequencies is not easy. Because a lot of research on this field has already been done we will not further discuss these problems. Rather, we will use the existing algorithms. Readers interested in radar parameter control are referred to e.g. [13].

\subsection{Re-planning issues}

In theory, the combination of these three stages should find the optimal sensor suite deployment in any environment for any mission. Dynamics in mission constraints, meteorological conditions combined with unexpected sensor performance prevent the system to always find optimal settings. When e.g., one of the sensors fails, all tasks assigned to that sensor need to be re-allocated to other and most likely less suited sensors. This means that some sensor tasks need to be dropped; in this case, the tasks directed at those objects that pose the lowest risk will be dropped first. Another complicating factor is the multi-platform setting is assigning tasks to sensors on different platforms. When that particular platform is forced to manoeuvre unexpectedly, the allocation process might have to start over. This becomes complex when the sensor suite is made up of sensors that deliver complementary data. In these cases the combination of tasks or the splitting of a task in two or more new tasks need to be taken into consideration as well.

A possible solution for this problem is to define several scenarios for which optimal solutions are calculated in the planning phase of a mission, these solutions are then called doctrines. The problem then is to define enough scenarios to cope with most situations and few enough to still be able to choose the right one in time. In each scenario, a part of the solution space is accurately modelled to enable finding good solutions in reasonable computing time.

Another solution is to use good exploration heuristics to find promising regions in the solution space. Within those regions exploitation heuristics can be used to find a good solution. In essence, this means that the space is not modelled in several scenarios but that we have a mechanism to choose from an unlimited set of scenarios. This approach is discussed by De Jong et.al. in [11]. The downside of this approach lies in the way the system can communicate with the user. Where scenarios are defined, the user can see which of those scenarios is currently active and alter that if desired. Explaining to the user what part of the solution space is currently examined is a challenging problem that may only be solved by demonstrating the system and gaining trust.

\section{INTEGRATION OF OPTICAL SENSORS}

In the previous section we showed that the uncertainties in the attributes of objects are input for the sensor tasks generation process. In case of kinematic information, for which radar systems are mostly deployed, the process accuracy can be estimated. But what should the system do about class uncertainty? How can we describe the difference in classification accuracy between an IR-sensor and a HD-sensor? This section tries to find the required building blocks to answer these questions.

\subsection{Desired contribution electro-optical sensor systems}

Optical sensors can contribute to the compilation of the OP in several ways: trainable optical sensors can improve the estimation of the position of an object by providing accurate azimuth and/or elevation angle information. The combination of this information with range information from laser range finder equipment or radar range information can significantly improve the determination of the 3 -D position of the object. 
Optical sensors are however especially useful in the reduction of the classification uncertainty and images provided by these types of sensors can be used (either by an operator or by a computer) to determine the class to which an object belongs.

The information enhancement may take the shape of an interaction between several $\mathrm{C} 2$ processes that use the OP as a mean of information exchange. If an object is tracked during a certain time interval e.g. by a mechanical target tracking radar or a Track While Scan (TWS) process, the kinematic behaviour of the object can be analysed to determine a preliminary object-class like 'ship' or 'aircraft'. This information can subsequently be used to 'tune' the detection algorithms described by T.Y.C. van Valkenburg-van Haarst et.al., [14]. After 'detecting' the object within the image, the part of the image that contains the object can be added to the OP-object as an attribute, allowing an operator to quickly inspect the visual characteristics of an object or, alternatively, the information can be handed over to an automatic classification process.

\subsection{Current use electro-optical sensor systems}

The current use of electro-optical sensor systems on board has many drawbacks. A human operator performs classification tasks, based on the expected objects and their silhouettes. This of course demands on a good view of the object in question. Section 1 already mentioned the decreasing knowledge, both in quantity and quality, that is available on board. Classification however has become increasingly complex due to the different types of mission and the advances in technology enabling higher speeds, thus reducing reaction time. This in turn stresses the operator into solving the classification problem in short time.

In order to be able to complete missions successfully, given the reduced the crew sizes and coping with the speeds of current and future technology, electro-optical sensor systems need to be integrated into the automatic sensor management process and the information provided by them has to be fused in the picture compilation process i.e. they are incorporated in the sensor control loop (Fig. 3) described by Bolderheij and Absil [15].

Currently, the main contribution of these sensors is desired and expected to be in the classification and identification process.

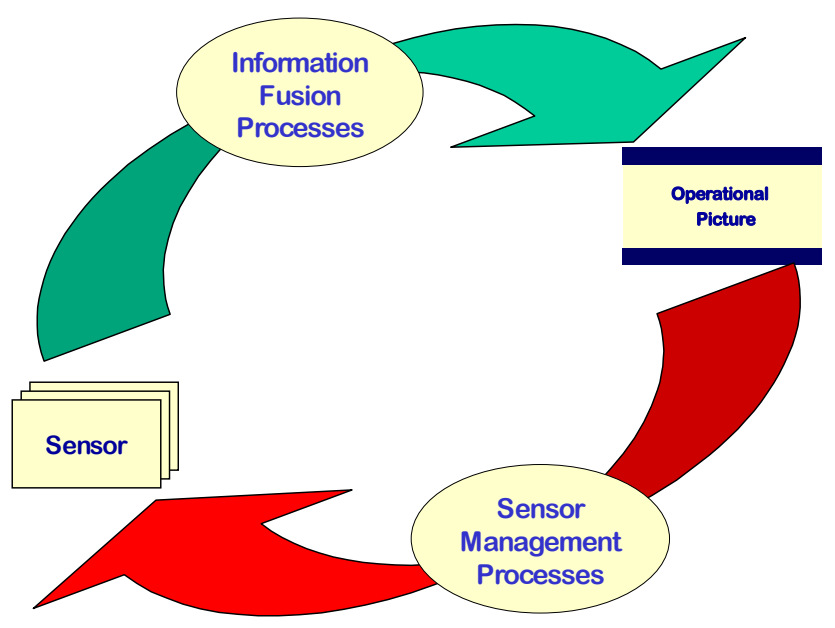

Fig. 3. Sensor Control Cycle

In order to find a good classification solution, several sub-processes have to done. Firstly, we need to know about the existence of the object. The object has to be distinguished from its surroundings. Before coming to more detailed classification labels, the domain (air or surface) has to be determined. Following this line, the lowest classification level is the objects' identity. 


\subsection{Algorithm demands}

For the Royal Netherlands Navy an automatic classification algorithm should be applicable in many different environments and for many different object types. The algorithm should be robust and highly reliable for variation in aspect angles and distances. Furthermore, the algorithm should be fast and should be able to generate a maximum reliable solution in a minimum amount of time depending on the available information. When the amount of information increases, the solution should adapt to a more detailed and more reliable classification of the object of interest. Or, when conflicting information becomes available, the solution should adapt by going back to more generic descriptions of the object.

The use of prior knowledge is expected to be helpful, but may not result in a system that only performs well on expected objects. The system has to be able to deal with unexpected objects. For example by addressing the operators' attention to the unexpected object, which can only be done when the system knows the object is unknown. Furthermore, the model has to be adaptive. This means that when more, or other, information becomes available that the solution of the classification algorithm will adapt. The solution may change or become more accurate.

For operational usability, the algorithm cannot be a hierarchical model. When certain information is not available the algorithm still has to produce a solution based on the available information. A general sketch of the resulting system is shown in Fig. 4.

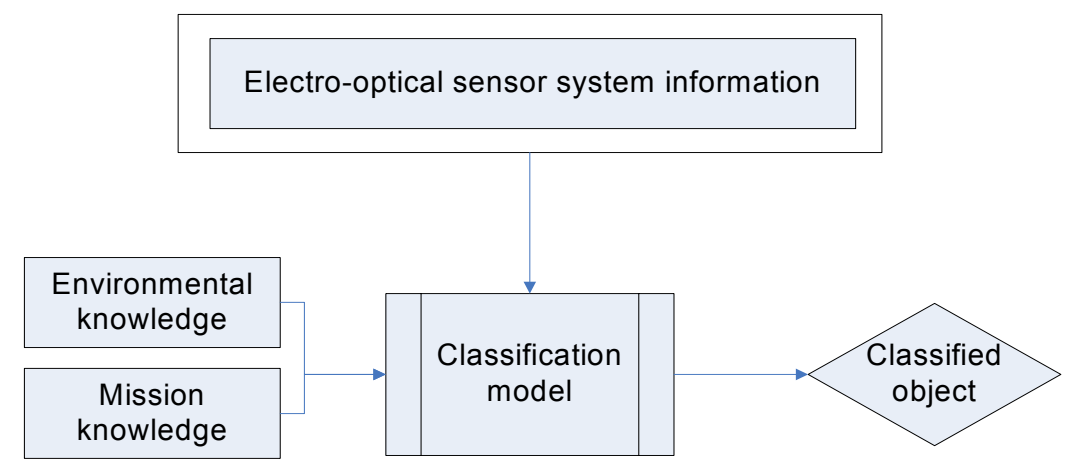

Fig. 4. Model contribution electro-optical sensor systems

\subsection{Research results with respect to detection}

In order to be able to classify an object based on electro-optical sensor information it is firstly desired to automatically detect the object in the images. To let the electro-optical sensor systems contribute to the automatic sensor management process, this research therefore focuses on the automatic detection of objects in a military, maritime environment. Since the subsequent goal is to classify the object, a bounding box is to be generated around the detected object.

Van Valkenburg-van Haarst et.al., [14] analyzed two different approaches for detecting objects in a military, maritime environment. Firstly, the position of the horizon is detected in order to pre-classify the object, knowing whether an object is a surface object or an air object, and to decrease the computation time of the detection algorithm. Furthermore, when surface threats are expected based on the mission, it can be an operational decision not to analyse the entire image but only the sea-part of the image. The position of the horizon is determined using gradient filters, which show to be well applicable for InfraRed (IR) images as well as for Visible Light (VL) images when the horizon appears as a sharp transition in the image [14].

For the actual object detection Van Valkenburg-van Haarst et.al., [14] analyzed two different approaches. The applicability of gradient filters as well as the use of polynomials for background estimation for object detection are analysed and tested on IR and VL images. The gradient approach for object detection focuses on the differences in intensity and localises areas with high gradients as detections. The polynomial background estimation approach uses the 
Linear Least Square Estimation (LLSE) in order to fit a line through the measured intensity values. The deviation between the fit and the actual measurements is used to detect objects in the image.

For the detection of objects the use of gradient filters shows poor performance. In the IR frequency band, as well as for VL images, much false detections occur, large objects are divided in several small objects and when a bounding box is generated it is improperly sized in most cases.

For the initial object detection the use of polynomials shows to perform well. In almost all IR and VL images the background estimation results in detection of the object. False detections do occur, but not as many as with the use of gradient filters by far. Furthermore, depending on the parameter settings larger objects are not subdivided into multiple small objects. However, the use of low-ordered polynomials does lead to improper box-sizes as well.

\subsection{Future research in EO image processing}

Since the main desired contribution of electro-optical sensor systems to the picture compilation process is automatic classification and identification, the generation of a properly sized bounding box around objects in the image is an important step. Therefore, and based on the results shown in [14], the combination of the polynomial background estimation method for the initial detection and the gradient approach for the detection of the actual edges will be investigated. Hereby, improvement of the clustering in order to decrease the number of objects that are split into several smaller objects, as well as the use of video streams in order to improve the performance with respect to the amount of false detections will be taken into consideration.

Furthermore, many of the parameter settings in both detection approaches are chosen arbitrarily. In order to show the applicability of a certain method this is assumed to be acceptable. However, in order to assure that the algorithms will work in an operational theatre where the objects and the geographical and meteorological circumstances are highly dynamic, the use of adaptive parameter settings is desired. Using adaptive parameter settings, the parameter values used will adapt to the meteorological and geographical circumstances as well as to the expected objects. Based on information of other sensor systems, as well as prior-knowledge of the environment and the mission, a proper choice of the parameter settings will be made. This means that the parameters that are now arbitrarily chosen will automatically be properly set by the mission information.

When the performance of the detection algorithms shows to perform robust and reliable enough to contribute to the automatic sensor management process, the classification algorithms will be developed. Currently, the idea is to investigate the applicability of the Recognition-By-Components (RBC) theory, which is one of the leading theories on how human beings classify objects in their environment. The RBC theory states that object recognition is achieved by assembling 3-dimensional geons ${ }^{1}$. As described by Biederman [16], the perceptual recognition of objects by human beings is conceptualized to be a process in which the image is segmented at regions of deep concavity into an arrangement of simple geometric components such as blocks, cylinders, wedges and cones. The fundamental assumption of the theory is that a modest set of generalized-cone components can be derived from contrasts of five readily detectable properties of edges in a two-dimensional image: curvature, collinearity ${ }^{2}$, symmetry, parallelism and cotermination. The detection of these properties is generally invariant over viewing position and image quality and consequently allows robust object perception when the image is projected from a novel view position or is degraded.

Due to the claimed robustness with respect to aspect angle, distance and image degradation the application of the recognition-by-components theory for automatic object recognition will be investigated.

\section{CONCLUSIONS AND FUTURE WORK}

The work presented in this paper describes an automated sensor management process where the operational picture is the central element. All decisions on sensor deployment are driven by the desire to make that picture as complete and accurate as possible relative to the mission at hand. We showed that sensor task requests can be generated automatically based on the current operational picture and the mission. Priorities can also be assigned to those requests to support planning and scheduling algorithms in allocating tasks to certain sensors.

1 Generalized-cone components

2 Lying on the same straight line 
When reducing uncertainty on the classification solution of object, one must be able to estimate the performance of electro-optical sensor systems. Furthermore, we showed that the support of or automation of the classification process based on images is necessary in the maritime military domain. The first step has been taken by developing algorithms to automatically detect the horizon in images so a distinction can be made between the air and surface domain. Also, algorithms were discussed that detect objects and find bounding boxes for those objects.

In the overall concept, the parameters of the mission are extremely important. It tells the system about expected targets, resulting in prior probabilities in reasoning processes. We can also set some meteorological default values specific to the mission to have a more accurate sensor performance prediction mechanism. In the future, we foresee that these factors will influence the parameters of the image processing. One could think of filter sizes dependent on target size and distance or specific polynomial orders given weather conditions.

When the automated classification based on images from electro-optical sensors is achieved, the generic three-stage sensor manager can be finished. For each of the objects' attributes tasks can be requested, and the performance of the different sensors can be estimated. Of course, future work then would be to implement these concepts in real systems and test them.

\section{REFERENCES}

[1] Strömberg D, Andersson M, Lantz F. On Platform-Based Sensor Management. Annapolis (ML): Proc. 5th Int. Conf. on Information Fusion; 2002, p 1374-80.

[2] McIntyre GA, Hintz KJ. A Comprehensive Approach to Sensor Management, Part I: A Survey of Modern Sensor Management Systems. IEEE Transactions on SMC April 1999.

[3] McIntyre GA, Hintz KJ. A Comprehensive Approach to Sensor Management, Part II: A new hierarchical model. IEEE Transactions on SMC April 1999.

[4] McIntyre GA, Hintz KJ. A Comprehensive Approach to Sensor Management, Part III: Goal Lattices. IEEE Transactions on SMC April 1999.

[5] Bolderheij F, van Genderen P. Mission Driven Sensor Management, Stockholm: Proc. 7th Int. Conf. on Information Fusion; 2004. p 799-804.

[6] Bolderheij F, Absil FG, van Genderen P. Risk-Based Object-Oriented Sensor Management, [CD-ROM]. Philadelphia: Proc. 8th Int. Conf. on Information Fusion; 2005.

[7] Thaens R. Sensor Scheduling Using Intelligent Agents, Stockholm: Proc. 7th Int. Conf. on Information Fusion. 2004. p 190-7.

[8] T'kindt V, Billaut J-C. Multicriteria Scheduling - Theory, Models and Algorithms, Springer Verlag, 2002

[9] van Norden WL, de Jong JL, Bolderheij F, Rothkrantz LJM. Intelligent Task Scheduling in Sensor Networks, [CDROM]. Philadelphia: Proc. 8th Int. Conf. on Information Fusion; 2005.

[10] Margialot M, Langholz G. 'Design and analysis of fuzzy schedulers using fuzzy Lyapunov', Engineering Applications of Artificial Intelligence, Vol. 14, No.2, p183-188, 2001.

[11] de Jong JL, van Norden WL. Application of hybrid metaheuristics in sensor management, Aerospace Science and Technology, Vol. 11, No.4, May 2007, p 295-302.

[12] van Norden, WL. 'Intelligent task scheduling in sensor networks: Introducing three new scheduling methodologies', Masters Thesis, Delft University of Technology, the Netherlands 2005. 
[13] Sabatini S, Tarantino M. 'Radar Parameter Design', Chapter 4 from Multifunction Array Radar - System Design and Analysis, Artech House, Norwood, USA 1994.

[14] van Valkenburg-van Haarst, TYC, Bolderheij F, Groen, FCA. "Automatic detection in a maritime environment: Gradient filter versus intensity background estimation", Proceeding of SPIE Defense and security conference, Orlando, 2008.

[15] Bolderheij F, Absil F.G.J. Mission-Oriented Sensor Management, Paris: Proc. Conf. on cognitive systems with interactive sensors; 2006.

[16] Biederman I. "Recognition-by-components: A theory of human image understanding", State University of New York at Buffalo, Copyright 1987 by the American Psychological Association, Psychological Review 1987, Vol 94, No.2, 115-147. 